\title{
Analysis of Multimode Interference Coupler With a Width of Arbitrary-Exponent Binomial Function
}

\author{
Hung-Chih Lu and Way-Seen Wang, Member, IEEE
}

\begin{abstract}
Multimode interference (MMI) couplers with a width of arbitrary-exponent binomial function are proposed. The proposed structure includes the conventional, butterfly, and parabolic structure such that the proposed MMI device can be used for equal and unequal power splitting. With a proper choice of the exponent of a binomial function, several equal and unequal optical powers splitting ratio can be obtained. For comparison, the optical power splitting ratios, total transmission, and coupler length of the proposed MMI couplers are discussed.
\end{abstract}

Index Terms-Binomial, coupler, multimode interference (MMI), power splitting.

\section{INTRODUCTION}

$\mathbf{O}$ PTICAL networks are now widely used in communication systems; the use of optical devices with good characteristics such as compact size, high fabrication tolerance, stable output, polarization independence, low power loss, etc., is then becoming more and more important. For these purposes, several optical devices such as wavelength division multiplexers, modulators, optical switches, and couplers have been proposed. In particular, multimode interference (MMI)-based couplers have been successfully applied in wavelength division multiplexers [1], modulators [2], optical switches [3], and couplers [4] due to their advantages of low loss, polarization independence, compact size, and low fabrication cost.

Recently, optoelectronic integrated circuits and modules are expected to be integrated on a single chip. For this reason, selectable power splitting ratio of devices is necessary in order to satisfy different varieties of applications. Several applications of MMI structure were then reported [4]-[6]. For example, MMI couplers with parabolic-tapered interference regions for equal power splitting [7]-[9] and with butterfly interference regions for unequal power splitting [10] were proposed. Obviously, the width functions of butterfly and the parabolic MMI region correspond to binomial functions of exponents 1 and 2, respectively. Therefore, an analysis of the dependence of the exponent of the binomial width function for MMI devices is necessary.

In this paper, arbitrary-exponent binomial MMI devices are presented. The device for equal and unequal power splitting are

Manuscript received February 15, 2007; revised May 15, 2007. This work was supported by the National Science Council, Taipei, Taiwan, R.O.C., under Contract 95-2221-E-002-326-MY3.

H.-C. Lu is with the Graduate Institute of Electro-Optical Engineering, National Taiwan University, Taipei 10617, Taiwan, R.O.C.

W.-S. Wang is with the Department of Electrical Engineering, National Taiwan University, Taipei 10617, Taiwan, R.O.C. (e-mail: wswang@cc.ee. ntu.edu.tw).

Digital Object Identifier 10.1109/JLT.2007.902765

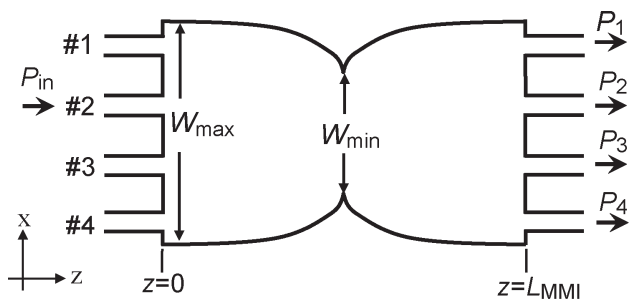

(a)

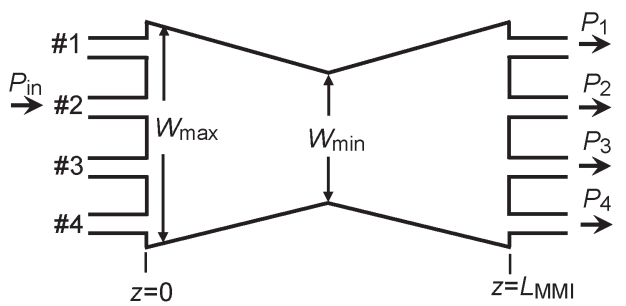

(b)

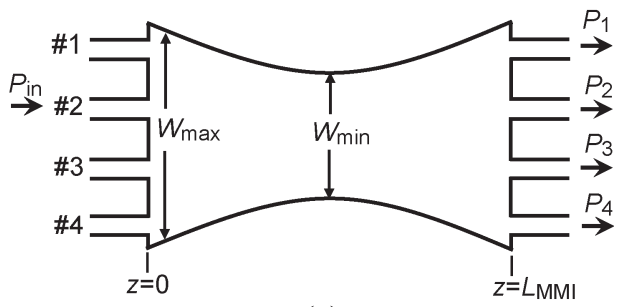

(c)

Fig. 1. Structure of arbitrary-exponent binomial MMI coupler. (a) $\sigma<1$. (b) $\sigma=1$. (c) $\sigma>1$.

designed by selecting a proper exponent for the width function. Moreover, the characteristics of arbitrary-exponent binomial MMI couplers are discussed.

\section{Proposed MMi Structure}

The proposed $4 \times 4$ arbitrary-exponent binomial MMI coupler is shown in Fig. 1, where the width function $W(z)$ is defined as given by

$$
W(z)=W_{\min }+\left(W_{\max }-W_{\min }\right) \cdot\left|1-2 \cdot \frac{z}{L_{\mathrm{MMI}}}\right|^{\sigma}
$$

where $W_{\max }$ and $W_{\min }$ are the maximum and the minimum width of the MMI region, $\sigma$ is a positive number, and $L_{\mathrm{MMI}}$ is the coupler length. Note that the values of $\sigma$ for conventional, butterfly, and parabolic MMI couplers are 0, 1, and 2, respectively. To find an approximate formula for calculating the 


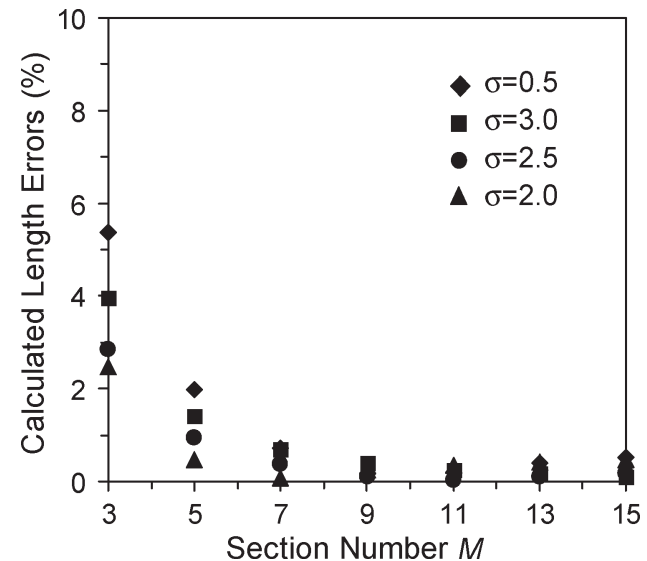

Fig. 2. Calculated length errors of the proposed $4 \times 4$ MMI couplers.

coupler length $L_{\mathrm{MMI}}$, the interference region is divided into $M$ sections to obtain

$L_{\mathrm{MMI}} \cong \frac{n_{\mathrm{g}}}{\lambda} / \sum_{i=1}^{M}\left\{\frac{1}{2 M} \cdot\left[W\left(\frac{i-1}{M}\right)^{-2}+W\left(\frac{i}{M}\right)^{-2}\right]\right\}$

where $n_{\mathrm{g}}$ and $\lambda$ are waveguide core index and signal wavelength, respectively. Note that the approximation formula (2) is dependent only on the sampling widths, so the calculation procedure is greatly simplified.

For comparison, $n_{\mathrm{g}}=3.3$ and $\lambda=1.507 \mu \mathrm{m}$ are chosen to simulate the proposed MMI device [7]. The waveguide cladding index is assumed to be 1. Furthermore, the proposed devices are simulated by the finite-difference beam propagation method (BPM). For convenience, the normalized width variation $d \Omega$ is defined as given by [7]

$$
d \Omega \equiv 1-W_{\min } / W_{\max } .
$$

For $W_{\max }=20 \mu \mathrm{m}$ and $W_{\min }=12 \mu \mathrm{m}$, the corresponding value $d \Omega$ is 0.4 . From those reported previously [7], the simulated transmissions are greater than $90 \%$, when $0 \leq d \Omega \leq 0.4$. As the MMI regions are slowly varying in width, a too large width difference $(d \Omega \geq 0.4)$ will lead to a higher transmission loss. The width of the access waveguides is $2.5 \mu \mathrm{m}$. The positions of four access waveguides are located at $x= \pm W_{\max } / 8$ and $\pm 3 W_{\max } / 8$.

Fig. 2 shows the lengths of the proposed $4 \times 4$ MMI couplers for $\sigma=0.5,2,2.5$, and 3 calculated by (2) and by BPM simulation for comparison. The grid and step size are 0.01 and $0.02 \mu \mathrm{m}$, respectively. It is obvious that the errors are less than $0.7 \%$ (or $3 \mu \mathrm{m}$ ) for $M \geq 7$. That indicates the proposed formula is accurate enough for practical application.

Fig. 3 shows that the coupler length $L_{\mathrm{MMI}}$ decreases with the increase of $\sigma$. As shown, the length reduction rate, defined as $-d L_{\mathrm{MMI}} / d \sigma$, becomes smaller when $\sigma$ is increased. That's because the variation of the width function is slower when $\sigma$ becomes large.

For a rigorous analysis, the power splitting ratio, defined as $P_{i} /\left(P_{1}+P_{2}+P_{3}+P_{4}\right)$, is calculated, where $P_{i}, i=$ $1,2, \ldots, 4$, are the output powers as indicated in Fig. 1 . The

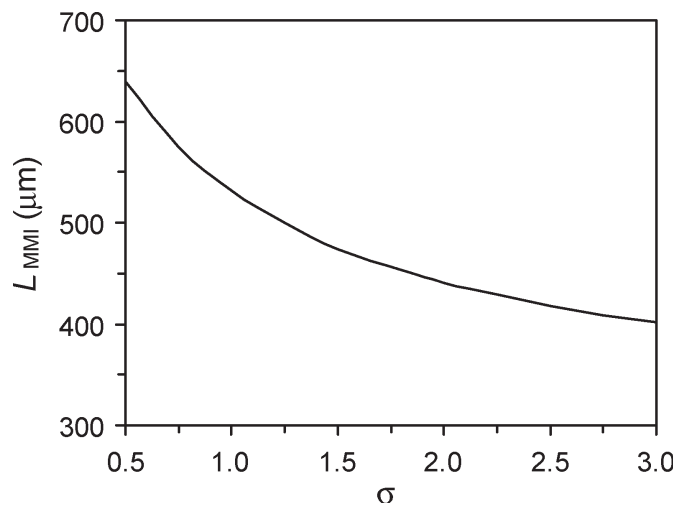

Fig. 3. $L_{\mathrm{MMI}}$ is influence by $\sigma$ of the proposed $4 \times 4 \mathrm{MMI}$ couplers for $d \Omega=0.4$

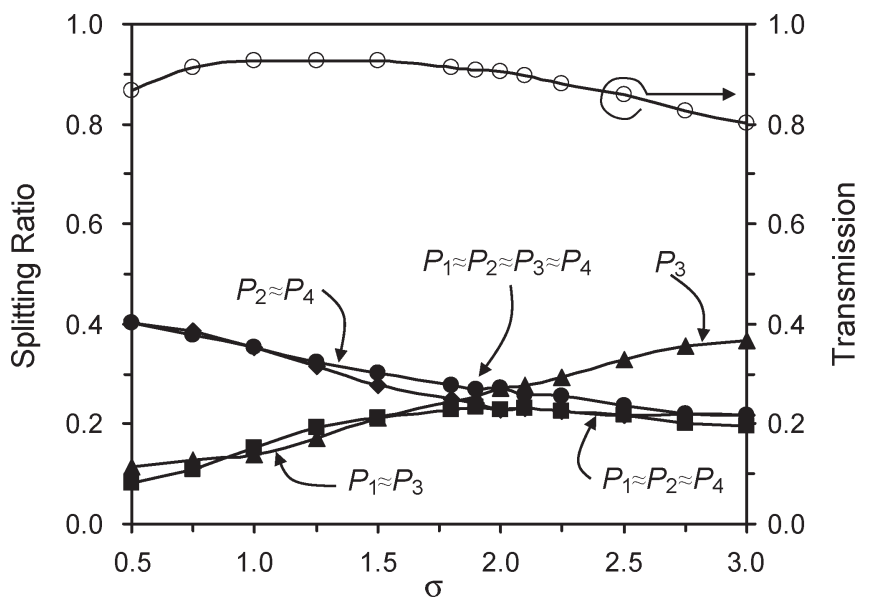

(a)

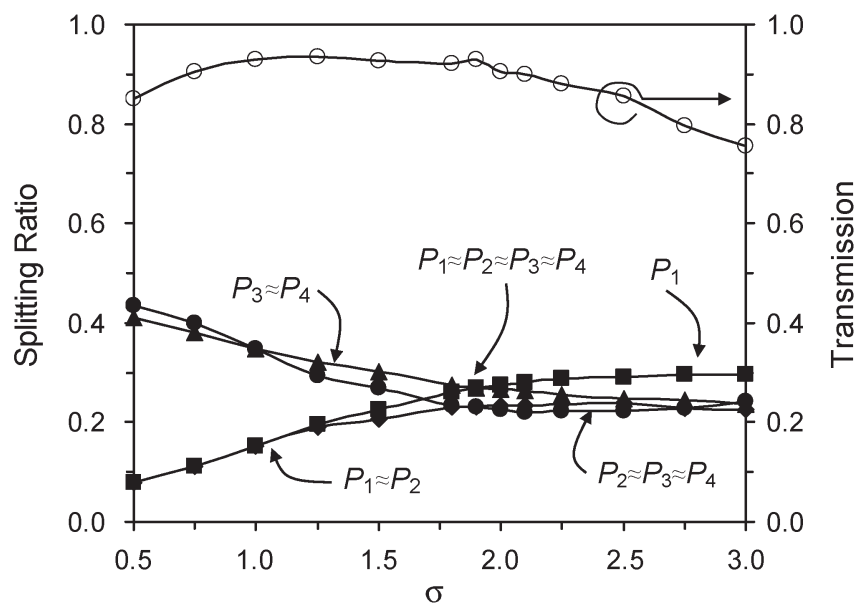

(b)

Fig. 4. Power splitting ratio is influence by $\sigma$ of the proposed $4 \times 4 \mathrm{MMI}$ couplers for $d \Omega=0.4$ with (a) inner input and (b) outer input.

power splitting ratio of the proposed $4 \times 4$ MMI couplers with $d \Omega=0.4$ versus the value of $\sigma$ is shown in Fig. 4(a). The input of MMI couplers chosen for simulation is $x= \pm W_{\max } / 8$. As shown from the figure, the power transmissions are all greater than $80 \%$ for $\sigma$ varying in the range of interest. In particular, the normalized output powers (or power splitting ratio) can be divided into three interesting cases, namely, dual-unequal power splitting (e.g., $P_{1} \approx P_{3}<P_{2} \approx P_{4}$ ), four-equal power 


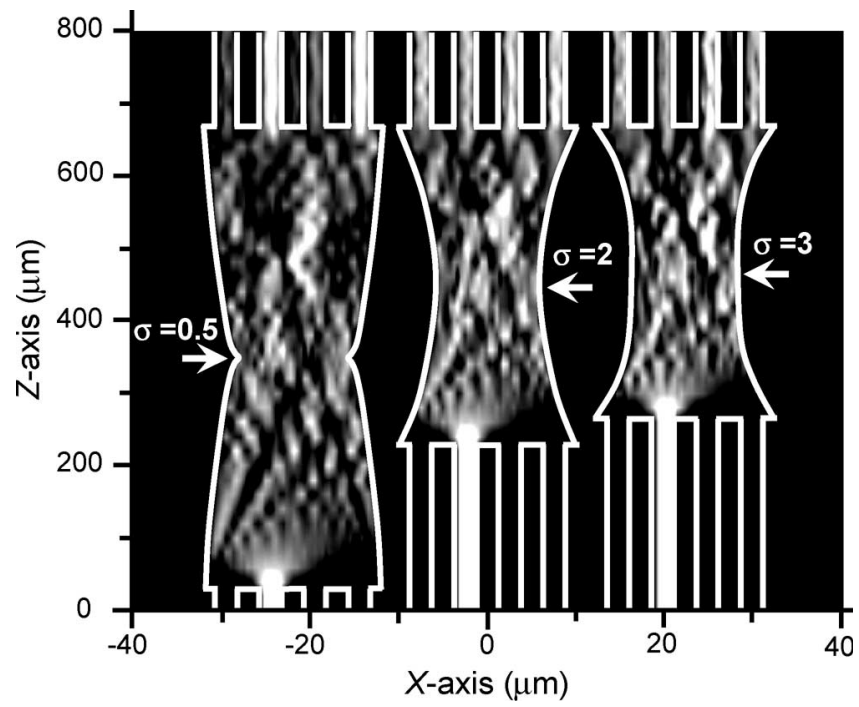

Fig. 5. Structure view and calculated intensity distribution of the proposed $4 \times 4$ MMI couplers for $\sigma=0.5,2$, and 3 .

splitting (e.g., $P_{1} \approx P_{2} \approx P_{3} \approx P_{4}$ ), and one-high-and-threeequal power splitting (e.g., $P_{1} \approx P_{2} \approx P_{4}<P_{3}$ ) for $0.5 \leq$ $\sigma<1.9,1.9 \leq \sigma \leq 2.1$, and $2.1<\sigma \leq 3$, respectively. The calculated intensity distributions of the proposed MMI couplers for $\sigma=0.5,2$, and 3 corresponding to the three power splitting cases are shown in Fig. 5. As shown from the figure, the shape and the length of the coupler are strongly dependent on the values of $\sigma$. Moreover, the distributions of the normalized output powers are quite different, although the laser accessing ports are the same. The details of the proposed MMI device for various types of power splitting are described as follows.

\section{A. Dual-Unequal Power Splitting}

Conventionally, MMI coupler for unequal-power splitting was implemented with a butterfly interference region $(\sigma=1)$. From Fig. 4(a), the maximum output power ratio, defined as $P_{\max } / P_{\min }$, for $\sigma=1$ is 2.55 , where $P_{\max }$ and $P_{\min }$ are the maximum and minimum output power, respectively. However, for $\sigma=0.5$, a larger maximum output power ratio of 4 can be obtained but with a lower transmission. Thus, a tradeoff value of $\sigma$ is chosen as 0.75 , which corresponds to a maximum output power ratio of 3.54. Obviously, for a similar power transmission, this power splitting ratio is greater than that for $\sigma=1$ (butterfly) [10]. Therefore, for a larger unequal-power splitting, $\sigma$ is better chosen as 0.75 rather than 1 . Note that the splitting ratio is dependent on both $\sigma$ and $d \Omega$.

With the chosen value of $\sigma$, the power splitting ratio, transmission, and $L_{\mathrm{MMI}}$ of the proposed $4 \times 4 \mathrm{MMI}$ couplers are calculated for different ratios of widths $(0 \leq d \Omega \leq 0.4)$, as shown in Fig. 6(a) and (b). As shown, the power transmission and $L_{\mathrm{MMI}}$ of the proposed MMI with $\sigma=0.75$ are very close to those of the butterfly $(\sigma=1)$. However, the output power ratios of the proposed MMI are higher than those of the butterfly MMI for various width ratios. It is clearly shown that the proposed MMI coupler for unequal power splitting with $\sigma=0.75$ is good for a reasonable wide range of width ratio.

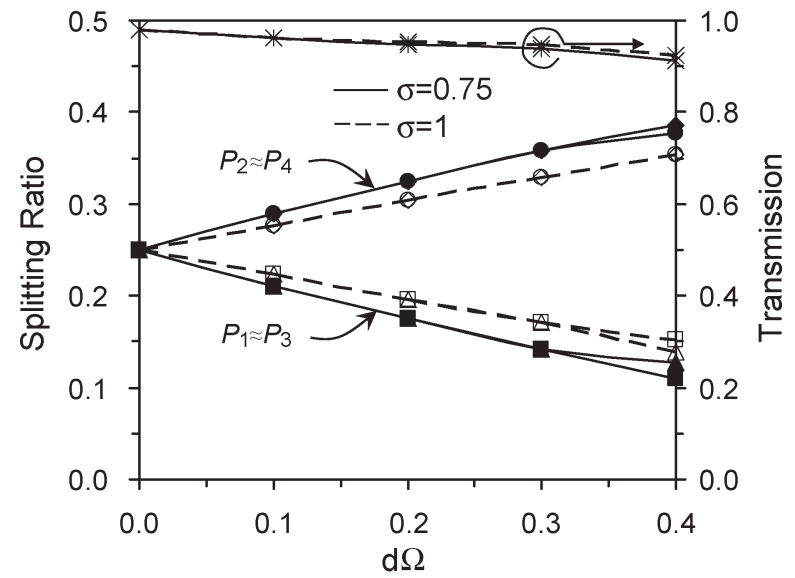

(a)

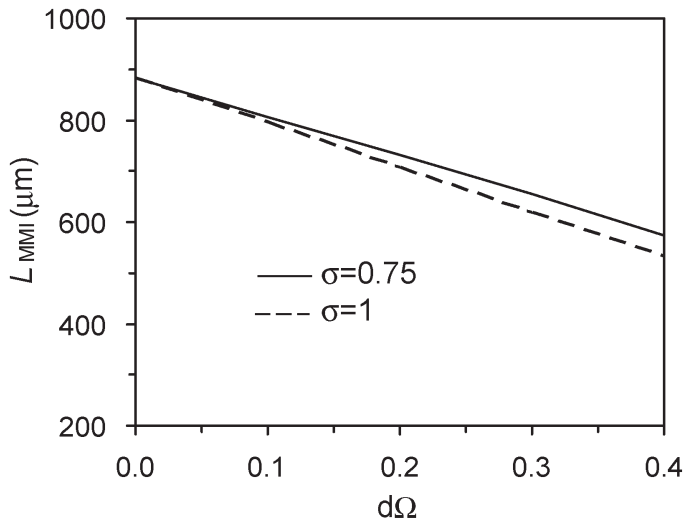

(b)

Fig. 6. Performance comparison of the proposed $4 \times 4$ MMI couplers for $\sigma=0.75$ and 1 (butterfly). (a) Splitting ratio. (b) $L_{\mathrm{MMI}}$.

\section{B. Four-Equal Power Splitting}

MMI coupler for equal-power splitting was implemented with a parabolic interference region $(\sigma=2)$. In this paper, the value of $\sigma$ is allowed to vary slightly from 1.9 to 2.1 for an appropriate choice. A comparison of power splitting ratio, transmission, and coupling length of the proposed $4 \times 4 \mathrm{MMI}$ couplers for $\sigma=1.9,2$, and 2.1 are shown in Fig. 7(a) and (b). As shown, the results for $\sigma=1.9-2.1$ are almost the same. That indicates the value of $\sigma$ is not very critical, which provides a tolerance for the device design.

\section{One-High-and-Three-Equal Power Splitting}

When $2.1<\sigma \leq 3$ is chosen for simulation, the power splitting becomes one with higher output power, but three with about the same lower power. Such an interesting power splitting ratio can be used for equally delivering optical power to three output ports, yet still have one remains enough power for subsequent ports located at longer distance. The splitting ratio, transmission, and $L_{\mathrm{MMI}}$ of the proposed $4 \times 4$ MMI couplers for $\sigma=3$ are shown in Fig. 8(a) and (b), respectively. The relative output powers are $P_{1} \approx 20 \%, P_{2} \approx 22 \%, P_{3} \approx 37 \%$, and $P_{4} \approx 22 \%$, respectively. The maximum output power ratio is increased from 1 to 1.87 when $d \Omega$ is varying from 0 to 0.4 . The total transmission is larger than $80 \%$. 


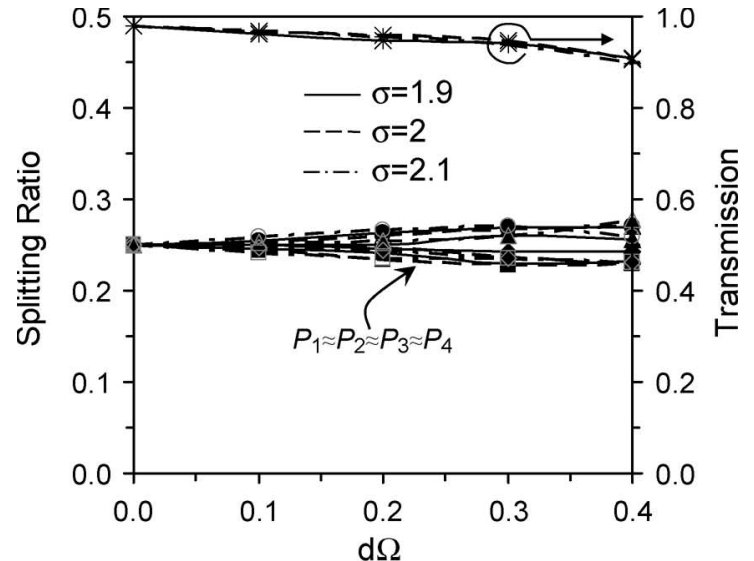

(a)

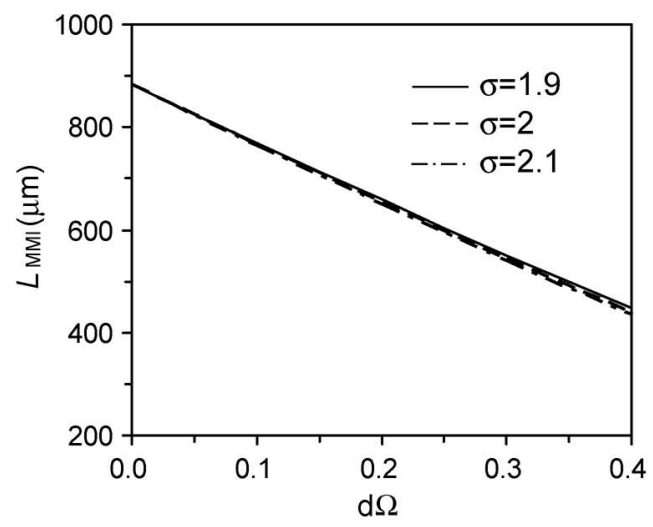

(b)

Fig. 7. Performance comparison of the proposed $4 \times 4$ MMI couplers for $\sigma=1.9,2$ (parabolic), and 2.1. (a) Splitting ratio. (b) $L_{\mathrm{MMI}}$.

Thus, various desired power splitting ratios of the proposed MMI coupler can be selected simply by choosing a suitable value of $\sigma$ for the width function.

\section{DISCUSSION}

It is known that the major influences of width variation of an MMI region are discontinuous phase change and intermodal coupling [7], [10]. For a width function with smaller value of $\sigma$ such as $0.5 \leq \sigma<1.9$, there are two kinks at the middle of the interference region. Guided waves propagate through the kink region encounter the discontinuity and undergo phase change. As the access location is a small offset to the central axis of the interference region, the modal powers are distributed unequally by the kink. By symmetry, two pairs of unequal-power splitting are formed. For example, assume the light is launched at the outer access port (port \#1 or port \#4 in Fig. 1), simulated results, as shown in Fig. 4(b), show that the output powers $P_{1}, P_{2}, P_{3}$, and $P_{4}$ satisfy the relation $P_{1} \approx P_{2}<P_{3} \approx P_{4}$. However, for light launching at the inner access port (port \#2 or port \#3 in Fig. 1), the output powers $P_{2}$ and $P_{3}$ are interchanged such that the previous output power relation becomes $P_{1} \approx P_{3}<P_{2} \approx$ $P_{4}$. Note that although the power splitting ratios are dependent on $\sigma$ and $d \Omega$, the relations $P_{1} \approx P_{2}<P_{3} \approx P_{4}$ and $P_{1} \approx P_{3}<$ $P_{2} \approx P_{4}$ are nearly unchanged.

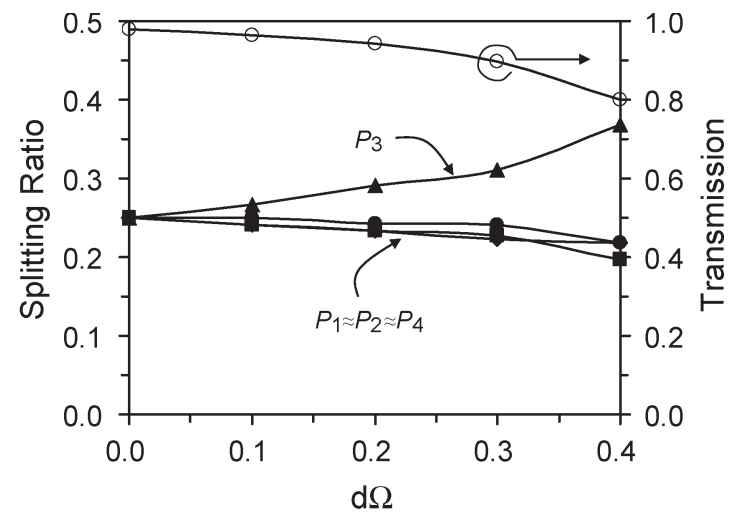

(a)

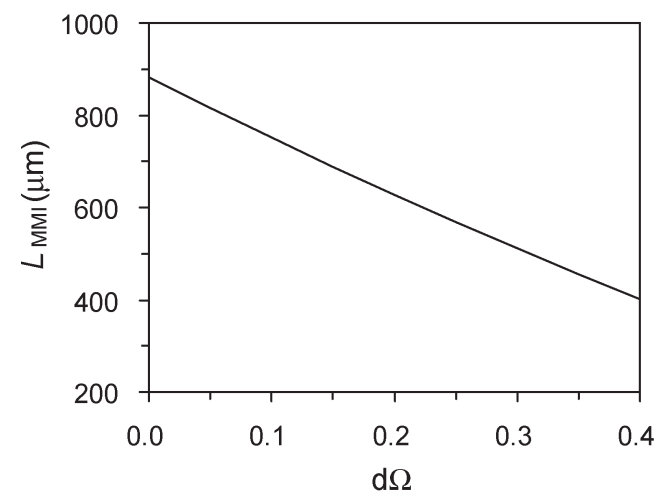

(b)

Fig. 8. Performance of the proposed $4 \times 4$ MMI couplers for $\sigma=3$. (a) Splitting ratio. (b) $L_{\mathrm{MMI}}$.

For the case of $1.9 \leq \sigma \leq 2.1$, the kinks at the middle of the interference region are not as sharp as those shown previously, so their effects can be neglected. Guided waves can then propagate through a smoothly curved interference region and hardly encounter any discontinuities for phase changes (i.e., $d W / d z=0$ at $z=L_{\mathrm{MMI}} / 2$ ). Moreover, as the normalized width variation is limited in the range $0 \leq d \Omega \leq 0.4$, the guided waves passing through the slowly varying interference region do not produce significant intermodal couplings. Thus, light launching at different access ports is not very critical to the output powers, and therefore, the relation of four-equal output powers $P_{1} \approx P_{2} \approx P_{3} \approx P_{4}$ can be achieved. Similarly, although the power splitting ratios are dependent on $\sigma$ and $d \Omega$, the relation $P_{1} \approx P_{2} \approx P_{3} \approx P_{4}$ remains nearly unchanged.

For the case of $2.1<\sigma \leq 3$, guided waves are propagating through an interference region where both ends are highly curved. Although the normalized width variation is limited in the range $0 \leq d \Omega \leq 0.4$, guided waves passing through the fast varying interference region generate significant intermodal coupling. Thus, the light access locations are critical to the output powers. More power is coupled to a favorable channel when the light is launched at port \#2 (or \#3), and therefore, the relation of one-high-and-three-equal power splitting $P_{1} \approx$ $P_{2} \approx P_{4}<P_{3}$, can be achieved. From the previous results, the maximum output power ratio $P_{3} / P_{1}$ is about 1.87 . In order to show the influence of intermodal coupling, access waveguides 
are tilted at an angle $\theta$. The angle can be derived from (1) by differentiation as given by [7]

$$
\theta \approx \tan ^{-1}\left(2 \sigma \cdot x \cdot d \Omega / L_{\mathrm{MMI}}\right) .
$$

When ports $\# 2$ and $\# 3$ are tilted at an angle of $0.86^{\circ}$, the maximum output power ratio is decreased to be 1.6. The relative output powers become $P_{1} \approx 21 \%, P_{2} \approx 22 \%, P_{3} \approx 34 \%$, and $P_{4} \approx 23 \%$, respectively. The total transmission power remains almost the same (i.e., 80\%). That shows titled inner access waveguides for a higher transmission are not needed. The only difference is the decreased power ratio.

Similarly, when the light is launching at port \#1 (or \#4) tilted at a larger angle of $2.56^{\circ}$, the one-high-and-three-equal power splitting relation can also be achieved. However, the relative output powers become $P_{1} \approx 29 \%, P_{2} \approx 23 \%, P_{3} \approx 25 \%$, and $P_{4} \approx 23 \%$, respectively. That means $P_{1}$ is now the largest, so the one-high-and-three-equal power splitting relation becomes $P_{2} \approx P_{3} \approx P_{4}<P_{1}$. The maximum output power ratio $P_{1} / P_{2}$ and the total power transmission are both decreased to be 1.27 and $73 \%$, respectively, due to intermodal coupling. When the access waveguides are not tilted, the total power transmission is $62 \%$, which is even smaller. That indicates the intermodal coupling is stronger, and therefore, tilted access waveguides are needed for outer channels to reduce the transmission loss.

Just the same as those mentioned previously, although the power splitting ratios are dependent on the $\sigma$ and $d \Omega$, the relation $P_{1} \approx P_{2} \approx P_{4}<P_{3}$ for light accessing at port \#2 (or \#3) and the relation $P_{2} \approx P_{3} \approx P_{4}<P_{1}$ for light source accessing at tilted port \#1 (or \#4) remain nearly unchanged.

It is also of interest to know when a width function with a value of $\sigma$ outside the range $0.5 \leq \sigma \leq 3$ discussed previously. For example, when $0.2 \leq \sigma<0.5$, the discontinuous phase changes are too large to have constructive interference. The transmission loss will be too large to be useful. Note that, when $0<\sigma<0.2$, the discontinuous phase change becomes smaller. From our simulation, the maximum output power ratio is reduced from 5.3 to 1.0 and the corresponding transmission is increased from $77 \%$ to $95 \%$ when $\sigma$ is varying from 0.2 down to 0 . However, the kinks in the interference region becomes very sharp, and therefore, the self-imaging behaviors of the proposed MMI for $0<\sigma<0.2$ is essentially the same as those of a rectangular one. Such a kinked interference region is not easily realizable and, therefore, is not considered. On the other hand, for $\sigma>3$, intermodal couplings are too strong to obey selfimaging. The transmission loss is also too large to be useful. Therefore, only when $0.5 \leq \sigma \leq 3$ is considered to be useful.

Moreover, for simplicity, only an interference region with the largest width variation of interest (i.e., $d \Omega=0.4$ ) is considered. Those interesting regions with a smaller value of $d \Omega$ are varying slower, and their transmission loss and maximum output power ratio are both decreased.

\section{CONCLUSION}

Arbitrary-exponent binomial MMI devices are proposed and analyzed. For a $4 \times 4$ arbitrary-exponent binomial MMI coupler, the splitting ratios can be divided into three interesting cases, namely, dual-unequal power splitting, four-equal power splitting, and one-high-and-three-equal power splitting simply by selecting a suitable exponent of the binomial function. As different varieties of width function such as sine function, cosine function, etc., of the MMI devices can be chosen for studying the power splitting ratios, it is expected that similar or even new behavior of power splitting can be seen. Moreover, when the number of input ports is greater than four, more cases of power splitting ratios can be obtained. Details of the application will be of interest for future studies.

\section{REFERENCES}

[1] M. R. Paiam and R. I. MacDonald, "Design of phased-array wavelength division multiplexers using multimode interference couplers," Appl. Opt., vol. 36, no. 21, pp. 5097-5108, Jul. 1997.

[2] S. R. Sakamoto, A. Jackson, and N. Dagli, "Substrate removed GaAs-AlGaAs electrooptic modulators," IEEE Photon. Technol. Lett., vol. 11, no. 10, pp. 1244-1246, Oct. 1999.

[3] N. S. Lagali, M. R. Paiam, R. I. MacDonald, K. Wörhoff, and A. Driessen, "Analysis of generalized Mach-Zehnder interferometers for variable-ratio power splitting and optimized switching," J. Lightw. Technol., vol. 17, no. 12, pp. 2542-2550, Dec. 1999.

[4] L. Soldano and E. Pennings, "Optical multi-mode interference devices based on self-imaging: Principles and applications," J. Lightw. Technol., vol. 13, no. 4, pp. 615-627, Apr. 1995.

[5] H.-C. Chien and R. V. Ramaswamy, "Symmetrical directional coupler as a wavelength multiplexer-demultiplexer: Theory and experiment," IEEE J. Quantum Electron., vol. 27, no. 3, pp. 567-574, Mar. 1991.

[6] J. Park, Y. Chung, S. Baek, and H.-J. Lee, "New design for low-loss star couplers and arrayed waveguide grating devices," IEEE Photon. Technol. Lett., vol. 14, no. 5, pp. 651-653, May 2002.

[7] D. Levy, R. Scarmozzino, and R. Osgood, "Length reduction of tapered $N \times N$ MMI devices," IEEE Photon. Technol. Lett., vol. 10, no. 6, pp. 830-832, Jun. 1998.

[8] D. Levy, R. Scarmozzino, Y. Li, and R. Osgood, "A new design for ultracompact multimode interference-based $2 \times 2$ couplers," IEEE Photon. Technol. Lett., vol. 10, no. 1, pp. 96-98, Jan. 1998.

[9] D. Levy, K. H. Park, R. Scarmozzino, and R. Osgood, "Fabrication of ultra-compact 3-dB $2 \times 2$ MMI splitters," IEEE Photon. Technol. Lett., vol. 11, no. 8, pp. 1009-1011, Aug. 1999.

[10] P. Besse, E. Gini, M. Bachmann, and H. Melchior, "New $2 \times 2$ and $1 \times 3$ multimode interference couplers with free selection of power splitting ratios," J. Lightw. Technol., vol. 14, no. 10, pp. 2286-2292, Oct. 1996.

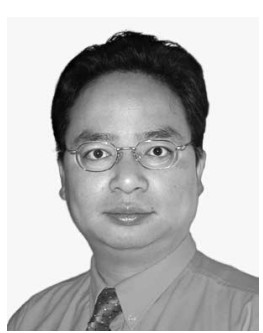

Hung-Chih Lu was born in Taoyuan County, Taiwan, R.O.C., on May 25, 1970. He received the B.S. degree in electronics engineering from Chung Yuan Christian University, Chung Li, Taiwan, in 1997, and the M.S. degree from National Taiwan University of Science and Technology, Taipei, Taiwan, in 1999. He is currently working toward the $\mathrm{Ph} . \mathrm{D}$. degree at the Graduate Institute of ElectroOptical Engineering, National Taiwan University, Taipei, Taiwan.

His current research interest includes the design of integrated optical waveguide devices and circuits.

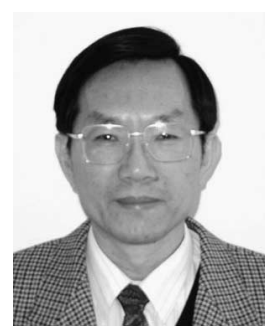

Way-Seen Wang (M'84) was born in Taipei, Taiwan, R.O.C., on March 11, 1948. He received the B.S. degree in electrical engineering from National Taiwan University, Taipei, in 1970, and the M.S. and $\mathrm{Ph} . \mathrm{D}$. degrees from the University of Southern California, Los Angeles, in 1975 and 1979, respectively.

Since August 1971, he has been with the Department of Electrical Engineering, National Taiwan University. In 1984, he became a Full Professor. His current research interest includes the design and fabrication of integrated optical waveguide devices. 